\title{
Associations between physical activity and health-related fitness: Differences across childhood
}

\author{
Zeinab KHODAVERDI 1, Jacqueline D. GOODWAY ${ }^{2}$, David F. STODDEN ${ }^{3}$ \\ ${ }^{1}$ Department of Motor Behavior, Faculty of Physical Education and Sport Sciences, Kharazmi University, Tehran, Iran. \\ 2 Department of Physical Activity and Educational Services, College of Education and Human Ecology, Ohio State University, Columbus, OH,USA. \\ ${ }^{3}$ Department of Physical Education \& Athletic Training, College of Education, University of South Carolina, Columbia, SC, USA. \\ Address correspondence to Z. Khodaverdi, e-mail: sara.khodaverdi@ymail.com
}

\begin{abstract}
The purpose of this study is to evaluate associations between physical activity (PA) intensities to aspects of health-related physical fitness (HRPF) across childhood (4-11 years). A sample of 160 children, including 4-5 years ( $\mathrm{n}=55)$, 7-8 years ( $\mathrm{n}=60)$, and 10-11 years $(\mathrm{n}=45)$ old, participated in this study. Five day PA levels (light, moderate, and vigorous) were measured using accelerometry. HRPF was assessed using curl-ups (abdominal strength/endurance), pushups (upper body strength/endurance), PACER (cardiorespiratory fitness) and grip strength. Hierarchical and linear multiple regression analysis quantified associations between HRPF components and PA levels across childhood. After controlling for BMI and age,_cardiorespiratory fitness accounted for 3\% of variation in vigorous in PA in 4-11 year-old children and significantly predicted $23 \%$ of variance in vigorous PA in late childhood. No other statistically significant associations between any aspect of fitness and any PA intensities were noted across age groups. These results support other research suggesting cardiorespiratory fitness may be the only aspects of HRPF that is related to various intensities of PA (as assessed via accelerometry), across childhood. An important implication of this study is that physical education should provide an equal emphasis on developing PA habits and HRPF as both are independently associated with health in childhood.
\end{abstract}

Keywords: Physical activity intensities, health, accelerometry, cardiorespiratory endurance, strength.

\section{INTRODUCTION}

Physical activity (PA) is a global descriptor of voluntary movement at various intensities that an individual performs (28), while physical fitness can be defined as the capacity to perform PA (25). Specifically, health-related physical fitness (HRPF) is defined by five components: cardiorespiratory fitness, muscular strength, muscle endurance, flexibility and body composition (8). While there is strong evidence linking both PA (17) and fitness (15) to health in youth, the relationship between the two constructs has been noted to generally be weak with correlations ranging from -0.16 to $+0.24(1,2,9,18,14,21,30)$. The generalized weak association between PA and HRPF in youth may be a function of the variability in measurement of PA/energy expenditure in children and adolescents across studies as well as potential differences in the strength of associations across age.

Limited evidence has addressed potential developmental differences across age when examining the relationship between PA and multiple components of HRPF. Katzmarzky et al. (18) in a cross-sectional study with a sample of 9-18 years old boys and girls, demonstrated that PA (measured by activity diaries) explained only $11 \%$ to $21 \%$ of the variance in healthrelated items (sit-ups, static leg strength, PWC150, skinfolds) (13). Though all canonical correlations were significant in most age groups, there were fluctuations in the strength of associations between each component of HRPF and moderate-to-vigorous PA and there was no clear trend in associations among HRPF components and PA across age groups. Aires et al., also followed changes in fitness (summed z-score of push-ups, curl-ups, $20 \mathrm{~m}$ shuttle run) and total PA 
(measured by questionnaire) for three years in a sample of 11-19 year-old adolescents (1). Changes in PA were directly and independently associated with changes in fitness in three consecutive years after adjustments for sex, age, and fitness at baseline. However, to our knowledge, no research has examined changes in the strength of associations among objectively measured PA and components of HRFP across childhood age groups (i.e., early, middle and late childhood). This study is significant as it addresses associations between PA and multiple aspects of fitness across three specific age groups across childhood. Additionally, while the majority of data suggest the relationship between aspects of fitness and PA (i.e., total or moderate-to-vigorous PA) is relatively weak in youth, differences in associations among different PA intensities levels (i.e., light, moderate, and vigorous) and aspects of fitness have not been addressed. Although the promotion of 60 minutes or more of daily PA focuses on moderate-to-vigorous PA for youth, the 2008 PA guidelines emphasize the health benefits of physical activities that typically are classified as vigorous (e.g., jumping rope) (35). Vigorous activities are important as continually high effort levels of PA in vigorous activities are demanded to produce consistent physiological changes associated with fitness improvement (35). Thus, it seems logical that higher fit children may be participating in more high intensity, or vigorous PA.

Alternatively, light physical activities also increase metabolic rate from baseline and contribute to total daily energy expenditure (26), especially in overweight/obese youth. It also is conceivable that daily light PA may have a protective effect against excess fat mass (9). While most studies that have examined associations between PA and HRPF have assessed total PA $(14,21,30)$ we noted only one study that has examined the relationship between aspects of fitness and different intensities of PA (2). Aires et al. examined associations between cardiorespiratory fitness (only one components of HRPF) and objectively measured PA intensities (light, moderate, moderate-tovigorous, vigorous, very vigorous) in adolescents aged 11-18 years old and found cardiorespiratory fitness was correlated only with vigorous PA (2). To our knowledge, no research has examined associations among all components of HRPF and various objectively measured PA intensities across younger children. The diversity in measurement approaches and analytical approaches across the literature makes it difficult to draw appropriate conclusions regarding strength of associations between various measures of HRPF and various intensities of PA across ages. Therefore, the purpose of this study was to examine associations between multiple components of HRPF and objectively measured light, moderate and vigorous PA levels in samples of children in early middle and late childhood.

\section{MATERIALS \& METHODS}

\section{Participants}

A convenient sample of youth ages $4-11$ years (93 boys, 67 girls; Mean $=7.68$ years, $S D=2.31$ ) in three categories including early childhood ( $4-5$ years; $n=55$, 28 boys and 27 girls), middle childhood (7-8 years; $\mathrm{n}=60,36$ boys and 24 girls), and late childhood (10-11 years; $n=45,29$ boys and 16 girls), were tested. We grouped children into three age groups for data analysis to assess the potentially different associations across age group. Children were recruited from two rural schools in the southwest US. The sample was 58\% Hispanic with the remaining participants being predominately non-Hispanic white. The majority of the children in the sample were classified as low socioeconomic status based on reduced lunch status. Prior to participation, approval from the schools districts and institutional human participants review board was obtained. Parental consent and child assent also were obtained. Youth with any physical disability or health condition that prevented completion of any of the fitness tests were not allowed to participate in testing. We conducted fitness testing using FITNESSGRAM (22) protocols for curl-ups (abdominal strength/endurance), pushups (upper body strength/endurance), and PACER (cardiorespiratory endurance) tests. We also included grip strength as a measure of total body strength. Previous research has demonstrated acceptable validity and reliability of these tests (22).

For younger children (4-5 years), we adapted the original PACER test protocols by having an adult run individually with children until the test ended. A recent study using 4-5 year old children (7) demonstrated high reliability for this adapted protocol $(r=.84, p<.001)$. It is important to note that formal testing using the FITNESSGRAM is not recommended until the fourth grade (22) as many younger children, 
specifically ages $4-5$, have difficulty in performing any pushups or curl-ups. A lack of experience, strength, and/or coordination and control in younger children may have influenced technical completion of pushup and curl-up tests. We examined grip strength using a children's hand grip dynamometer (Lafayette Instrument, Lafayette IN). The best score of three trials for each hand was averaged and used for data analysis (6).

Five-day daily PA levels were assessed using Actigraph accelerometers (Pensacola, FL). The Actigraph accelerometer has been validated for use in children and even children as young as preschool (29). Children were instructed to wear the accelerometers, which were attached by an elastic belt on the right hip during waking hours, with the exception of time spent bathing and engaging in other activities involving water. Children wore accelerometers for a minimum of five days ( 3 week and 2 weekend days) and were set at 15-second epochs. Counts were classified into light, moderate, and vigorous categories using empirically based cut points (10). Evenson et al.'s (10) ActiGraph cut points are recommended to estimate time spent in sedentary, light, moderate, and vigorous physical intensity activity in children and adolescents (33). Acceptable inclusion criterion for wear time was at least nine hours per day (27).

\section{Procedures}

Children completed a general warm-up routine before any HRPF testing. Height and mass of participants were measured using a physician's scale. Values to the nearest $0.5 \mathrm{~cm}$ and $100 \mathrm{~g}$ were recorded and these data used to calculate body mass index [BMI = weight $(\mathrm{kg}) /$ height $\left.\left(\mathrm{m}^{2}\right)\right]$. Trained research staff tested younger children (ages 4-5) individually on pushups, curl-ups, grip strength and the PACER test. Children ages 7-11 were tested in groups of two or three. Instruction and modeling for all children were provided on curl-ups, pushups, grip strength and the PACER test. Children also were prompted to demonstrate correct technique, if possible, on curl-ups and pushups (one or two trials) before the actual testing. Fitness tests were performed in a school gymnasium during their normal physical education time. In general, participants completed the HRPF tests during two testing sessions. Children completed the PACER test on a separate day from all other fitness tests. Compliance with wearing accelerometers was facilitated by guidelines previously published (34) and included: 1) a letter to the parents explaining placement of the accelerometer and by providing a simple diagram, 2) the use of parent-teacher conference nights to physically show the parent how to place the accelerometer, 3) a smiley face on accelerometer and belt to indicate right side up, 4) training in physical education classes to fit the belt and practice wearing it prior to formal data collection, 5) phone calls at home on the weekend to prompt wearing the belt, 6) checking placement of accelerometers in the children's home room every morning before classes started, and, 7) a sticker reinforcement chart to promote daily wearing of the belt to school.

\section{Statistical Analysis}

Three hierarchical multiple regressions were conducted to examine associations between fitness components and intensities of PA (light, moderate, and vigorous). Prior to conducing a hierarchical multiple regression, the relevant assumptions of this statistical analysis were tested. Firstly, a sample size of 160 was deemed adequate given 6 independent variables (age, BMI, curl-up, push-up, PACER, and hand grip) to be included in the analysis (32). The assumption of singularity was also met as the independent variables were not a combination of other independent variables. Residual and scatter plots indicated the assumptions of normality, linearity and homoscedasticity were all satisfied in entire sample (12). Age and BMI were entered at stage one of each regression as independent control variables. Cardiorespiratory endurance (PACER) was entered in stage two. Since muscular components (curl-up, push-up, and hand grip) measure health-related component differs from cardiorespiratory fitness, they all entered as independent variables at stage three to examine how they induce variance in PA above and beyond the cardiorespiratory fitness. Follow-up linear regressions by age group were run to examine predictors of the different PA intensities in each age group, separately if age was a significant predictor of hierarchical multiple regressions at stage three. Totally, a three-staged hierarchical multiple regression for each PA intensities (light, moderate, and vigorous) and three linear multiple regressions for each PA intensity in three age groups (4-5, 7-8, and 10-11 years) were conducted. Data analysis was carried out via SPSS version 20.0 for 
Windows (Chicago, IL, US). Statistical significance was set at alpha levels less than 0.05 .

\section{RESULTS}

Table 1 shows subjects' descriptive characteristics of age, fitness and PA levels across the three age groups. All measures of HRPF demonstrated higher average performances across age groups. PA levels did not follow that same trend. The 10-11 year-old group had the highest average LPA and that was followed by the $4-5$ year-old group. The $4-5$ year-old group and 7-8 years-old group had highest MPA and VPA, respectively. Correlations between variables are reported in Table 2 and the regression statistics are reported in Table 3.

The hierarchical multiple regression revealed that at stage one, age and BMI contributed significantly to the regression model, $F(2,157)=11.82, p<.001)$ and accounted for $13 \%$ of the variation in VPA. Introducing the cardiorespiratory fitness variables explained an additional $3 \%$ of variation in VPA and this change in $\mathrm{R}^{2}$ was significant, $\mathrm{F}(1,156)=6.72, p<.05$. BMI was no longer a significant predictor of VPA when cardiorespiratory fitness was added in stage two. Adding muscular components in stage three did not explain any additional variance in VPA. When all six independent variables were included in stage three of the regression model, age and cardiorespiratory fitness were significant predictors of VPA and accounted for $17 \%$ of the variance in VPA, F $(6,153)=5.23, p<.001$. Given the significant contribution of age and cardiorespiratory endurance as predictors of VPA, results of the follow-up linear regressions run by age group (4-5, 7-8, and 10-11 years old) showed that cardiorespiratory fitness was the only factor of HRPF significantly associated with VPA and accounted for $23 \%$ variance in VPA $(\beta=.39, p<.05)$ in late childhood only. No other significant results were found between any component of HRPF and any intensity of PA across age groups.

Table 1. Descriptive data and row scores $(n=160$; Mean \pm SD).

\begin{tabular}{|c|c|c|c|c|}
\hline Variables & $4-5$ years old $(n=55)$ & $7-8$ years old $(n=60)$ & $10-11$ years old $(n=45)$ & Total $(n=160)$ \\
\hline Age (year) & $4.96 \pm 0.55$ & $7.96 \pm 0.59$ & $10.63 \pm 0.43$ & $7.68 \pm 2.31$ \\
\hline BMI $\left(\mathrm{kg} / \mathrm{m}^{2}\right)$ & $16.11 \pm 2.48$ & $18.26 \pm 4.09$ & $21.97 \pm 6.16$ & $18.57 \pm 4.92$ \\
\hline Hang Grip & $9.51 \pm 3.11$ & $16.98 \pm 2.50$ & $22.22 \pm 3.93$ & $15.89 \pm 5.97$ \\
\hline Pacer (S) & $7.80 \pm 3.26$ & $17.28 \pm 8.32$ & $26.48 \pm 12.32$ & $16.61 \pm 11.22$ \\
\hline Push-ups (count) & $1.98 \pm 3.36$ & $7.21 \pm 7.09$ & $8.11 \pm 8.38$ & $5.66 \pm 7.02$ \\
\hline Curl-ups (count) & $2.22 \pm 3.38$ & $18.13 \pm 19.72$ & $32.17 \pm 21.96$ & $16.61 \pm 20.56$ \\
\hline Ave LPA (min/day) & $156.20 \pm 57.56$ & $146.42 \pm 32.82$ & $163.35 \pm 63.01$ & $154.55 \pm 51.68$ \\
\hline Ave MPA (min/day) & $42.21 \pm 17.80$ & $25.14 \pm 6.95$ & $24.18 \pm 12.07$ & $30.83 \pm 15.49$ \\
\hline Ave VPA (min/day) & $16.16 \pm 7.29$ & $27.77 \pm 4.87$ & $10.78 \pm 8.04$ & $13.41 \pm 7.27$ \\
\hline
\end{tabular}

BMI, body mass index; LPA, light physical activity; MPA, moderate physical activity; VPA, vigorous physical activity; SD, standard deviation; S, second; Ave, average.

\section{DISCUSSION}

The purpose of this study was to evaluate associations between PA intensities, assessed by accelerometer, and aspects of HRPF across early (4-5), middle (7-8) and late (10-11) childhood. The main finding of this study was that after controlling for BMI and age, cardiorespiratory fitness accounted 3\% of variation in vigorous in PA in 4-11 years old children and, more notably, $23 \%$ of variance in vigorous PA late childhood. No other significant associations between any intensity of PA with any aspect of HRPF were found. While this result is consistent with the general findings of previous literature, these data provide more direct evidence on the relationship between multiple measures of HRPF and objectively assessed PA across childhood age groups. These results agree with a number of studies, specifically in late childhood 
(e. g., 18) that reported a weak to moderate association between PA and cardiorespiratory fitness. Indeed, youth that engage in more intense exercise are more likely to improve their cardiorespiratory fitness (11) and sustain intense activity for a longer time. In addition, the alignment of the measurement device with the mode of fitness assessment (i.e., cardiorespiratory endurance via running) may also provide an understanding of why cardiorespiratory fitness is the most consistent correlate with accelerometry. This result also may be due in part to differences in age groups via and growth-related changes that occur in late childhood as the age range of 10-12 years is the time frame where maximal aerobic power $\left(\mathrm{VO}_{2}\right.$ max $)$ experiences an increase in most children (19). It was somewhat surprising that no other statistically significant associations were found between any HRPF test and vigorous PA levels. This speaks to the relative independence between aspects of HRPF and PA, specifically as they change across childhood (4,21). For example, Malina (21) did not find an association between PA and muscle strength or body composition, even though one of the outcomes suggested to be developed with PA across childhood is muscle strength (21). This result may indicate that other types of stimulus (e.g. specific types of resistance training) are needed in order to improve muscular strength (36). Overall, the relationship of PA with muscular strength is poorly understood especially in young people and more research is warranted.

Table 2. Correlations among variables across age-groups.

\begin{tabular}{|c|c|c|c|c|c|c|c|c|}
\hline Variables & BMI & Handgrip & PACER & Push-ups & Curl-ups & AveLPA & AveMPA & AveVPA \\
\hline \multicolumn{9}{|l|}{ Age } \\
\hline 4-11 yrs. & $.470^{* *}$ & $.865^{* *}$ & $.670^{* *}$ & $.374^{* *}$ & $.615^{* *}$ & .035 & $-.482^{* *}$ & $-.289^{* *}$ \\
\hline \multicolumn{9}{|l|}{ BMI } \\
\hline 4-5 yrs. & & -.003 & -.149 & -.050 & -.185 & .031 & .200 & -.085 \\
\hline 7-8 yrs. & & .340 & -.400 & -.429 & -.187 & .168 & .009 & -.227 \\
\hline 10-11 yrs. & & $.532^{* *}$ & $-.552^{* *}$ & $-.452^{* *}$ & -.182 & -.180 & -.141 & $-.322^{*}$ \\
\hline 4-11 yrs. & & $.552^{* *}$ & -.003 & $-.171^{*}$ & .139 & -.006 & $-.203^{*}$ & $-.328^{* *}$ \\
\hline \multicolumn{9}{|l|}{ Handgrip } \\
\hline $4-5$ yrs. & -.003 & & $.578^{* *}$ & $.361^{* *}$ & $.437^{* *}$ & -.115 & -.144 & .049 \\
\hline 7-8 yrs. & .340 & & .085 & -.060 & .161 & .134 & .123 & .061 \\
\hline 10-11 yrs. & .532 & & -.086 & .053 & .006 & -.125 & -.046 & -.194 \\
\hline 4-11 yrs. & $.552^{* *}$ & & $.580^{* *}$ & $.346^{* *}$ & $.528^{* *}$ & -.009 & $-.459^{* *}$ & $-.276^{* *}$ \\
\hline \multicolumn{9}{|l|}{ PACER } \\
\hline $4-5$ yrs. & -.149 & $.578^{* *}$ & & $.471^{* *}$ & $.578^{* *}$ & -.125 & -.169 & .124 \\
\hline 7-8 yrs. & -.400 & .085 & & .430 & .403 & -.130 & .106 & .221 \\
\hline $10-11$ yrs. & -.552 & -.086 & & $.768^{* *}$ & -.027 & .051 & .279 & $.422^{* *}$ \\
\hline 4-11 yrs. & -.003 & $.580^{* *}$ & & $.655^{* *}$ & $.487^{* *}$ & .013 & $-.260^{* *}$ & .008 \\
\hline \multicolumn{9}{|l|}{ Push-ups } \\
\hline 4-5 yrs. & -.050 & $.361^{* *}$ & $.471^{* *}$ & & $.515^{* *}$ & .100 & .043 & .160 \\
\hline $7-8$ yrs. & -.429 & -.060 & .430 & & .260 & -.106 & .118 & .275 \\
\hline 10-11 yrs. & -.452 & .053 & .768 & & .148 & .116 & .173 & .231 \\
\hline 4-11 yrs. & $-.171^{*}$ & $.346^{* *}$ & $.655^{* *}$ & & $.367^{* *}$ & .038 & -.137 & .081 \\
\hline \multicolumn{9}{|l|}{ Curl-ups } \\
\hline 4-5 yrs. & -.185 & $.437^{* *}$ & $.578^{* *}$ & $.515^{* *}$ & & -.033 & -.070 & -.014 \\
\hline $7-8$ yrs. & -.187 & .161 & .403 & .260 & & -.195 & .057 & .160 \\
\hline 10-11 yrs. & -.182 & .006 & -.027 & .148 & & -.042 & -.034 & .073 \\
\hline 4-11 yrs. & .139 & $.528^{* *}$ & $.487^{* *}$ & $.367^{* *}$ & & -.037 & $-.288^{* *}$ & -.103 \\
\hline
\end{tabular}

${ }^{*} \mathrm{P}<.05 ;{ }^{* *} \mathrm{P}<.01$ 
Table 3. Summary of hierarchical regression analysis for variables predicting VPA $(\mathrm{n}=160)$.

\begin{tabular}{llllc}
\hline Variable & $\mathrm{R}^{2}$ & $\Delta \mathrm{R}^{2}$ & $\mathrm{~b}$ & $\mathrm{t}$ \\
\hline Step one & $.13^{*}$ & $.13^{*}$ & & \\
Age & & & $-.54^{*}$ & $-2.05^{*}$ \\
BMI & & & $-.36^{*}$ & $-2.92^{*}$ \\
Step two & $.16^{*}$ & $.03^{*}$ & & \\
Age & & & $-1.33^{*}$ & $-3.33^{*}$ \\
BMI & & -.18 & -1.34 \\
PACER & & $.18^{*}$ & $2.59^{*}$ \\
Step three & .17 & .004 & & \\
Age & & & $-1.3^{*}$ & $-2.44^{*}$ \\
BMI & & -.14 & -.89 \\
PACER & & $.17^{*}$ & $2.04^{*}$ \\
Handgrip & & & -.06 & -.32 \\
Push-up & & .05 & .48 \\
Curl-up & & & .01 & .55 \\
\hline * p <.05. & & & & \\
& & & & \\
\hline
\end{tabular}

Findings of this study also did not show any other significant associations among aspects of HRPF and other intensities of PA. This may be due to a lack of agreement in cut-off points used to define PA with accelerometer. However, we used established cut point classifications acceptable for all levels of PA in children and adolescents (33). Indeed, there is a lack of consensus with regard to the cut-off points applied to define intensity levels in youth, which can lead to differences in the amounts of time spent in each PA level depending on the criterion selected to distinguish different intensities of PA (3).

A strength of this study was the use of accelerometers to assess different intensities of PA. The use of an objective PA measure with a high compliance rate (at least 9 hours/day) enhances the confidence in our findings because it was suggested that objective measures such as accelerometers provide more valid assessments for youth of all ages (37). Moreover, this study methodology specifically addressed potential differences across three childhood age ranges (i.e., early, middle, and late childhood). However, in light of findings from this study, some methodological issues should be considered. One potential consideration is associated with differences in estimating vigorous PA according to epoch time (sampling interval) procedures. Some studies point out that a shorter epoch (5 or 1 seconds) would be more sensitive to assess PA than the epoch used in this study (15 seconds), specifically for vigorous PA in young children as they demonstrate more short bursts of PA (24). In addition, the construct validity of HRPF assessments in early and middle childhood may not be as strong as in late childhood due to various issues including a lack of experience (i.e., coordination and control) in specific multi-joint movements (e.g., pushups and curl-ups) as well as potential cognitive capability and motivation issues related to completing the tests with maximum effort (15). However, a child's capability to complete the HRPF tests at various levels demonstrates their functional capability to control and coordinate their body mass in various types of real-life movements. Thus, these types of field-based tests (along with other developmentally appropriate movements) are important for children to be able to demonstrate across childhood (23). Although the relationship between PA and HRPF in youth may be confounded by physical development and maturational changes (20), we could not assess potential effects of maturation as these data were not available. Although this study compared associations across different age groups; longitudinal tracking of HPRF and PA would provide more specific information regarding changes in the strength of associations across childhood.

This study demonstrates the relative independence of PA behaviors and HRPF levels across specific ages in childhood. Based on results of this study and others, as well as the fact that PA and HRPF are independently associated with health markers and outcomes, it seems logical that childhood interventions should emphasize both PA and aspects of HRPF to meet the goal of at least $60 \mathrm{~min}$ moderate to vigorous/vigorous PA per day and healthy fitness levels in childhood. However, since recent metaanalyses suggest childhood focused PA and fitness interventions have largely been either unsuccessful or only minimally impactful in producing sustained increases in PA or fitness (31) developing and implementing intervention strategies with a developmental focus may be warranted. There is strong evidence showing a positive relationship between the development of motor competence and different intensities of PA and multiple aspects of 
HRPF across childhood and into adolescence (e.g., 16). Thus, it may be important to promote context specific, developmentally appropriate physical activities that enhance a child's capability to successfully navigate various PA environments and enhance aspects of fitness data indicates these integrated aspects of child development, along with aspects of self-concept, synergistically and recursively interact across childhood (e.g., 5). Thus, a comprehensive approach for developing and sustaining health-enhancing PA and HRPF across childhood may prove to be more successful than addressing them individually.

\section{Applicable Remarks}

- This is the first study to examine associations between objectively assessed physical activity and multiple components of health-related physical fitness across childhood.

- Data generally agrees with previous data which cardiorespiratory fitness is only related to vigorous physical activity.

- No other associations noted between physical activity and health-related physical fitness.

- This study highlights potential need to address physical activity and health-related physical fitness separately to promote health.

\section{REFERENCES}

1. Aires L, Anderson LB, Mendonca D, Silva G, Mota J. A 3-year longitudinal analysis of changes in fitness, physical activity, fatness and screen time. Acta Paediatrica, 2010; 99(1): 140-144.

2. Aires L, Silva P, Silva G, Santos MP, Ribeiro JC, Mota J. Intensity of physical activity, cardiorespiratory fitness, and body mass index in youth. Journal of Physical Activity and Health, 2010; 7: 54-59.

3. Anderson $C B$, Hagstromer $M$, Yngve A. Validation of the PDPAR as an adolescent diary: Effect of accelerometer cut points. Medicine \& Science in Sports \& Exercise, 2005; 37(7): 1224-1230.

4. Armstrong N, Williams J, Balding J, Gentle P, Kirby B. The peak oxygen uptake of British children with reference to age, sex and sexual maturity. European Journal of Applied Physiology, 1991; 62(5):369-75.

5. Babic MJ, Morgan P J, Plotnikoff RC, Lonsdale C, White RL, Lubans DR. Physical activity and physical self-concept in youth: Systematic review and meta-analysis. Sports Medicine, 2014; 44:1589-1601.

6. Baumgartner T, Jackson A, Mahar M, Rowe D. Measurement for evaluation in physical education \& exercise sciences. New York, NY: McGraw Hill, 2003.
7. Bürgi F, Meyer U, Granacher U, Schindler C, Marques-Vidal P, Kriemler S, Puder JJ. Relationship of physical activity with motor skills, aerobic fitness and body fat in preschool children: A crosssectional and longitudinal study (Ballabeina). International Journal of Obesity, 2011; 35: 937- 44.

8. Caspersen CJ, Powell KE. Christenson GM. Physical activity, exercise, and physical fitness: Definitions and distinctions for health-related research. Public Health Reports, 1985; 100: 126131.

9. Ekelund U, Aman J, Yngve A, Renman C, Westerterp K, Sjöström M. Physical activity but not energy expenditure is reduced in obese adolescents: a case-control study. American Journal of Clinical Nutrition, 2002; 76(5): 935-41.

10. Evenson KR, Cattellier D, Gill K, Ondrak K, McMurray RG. Calibration of two objective measures of physical activity for children. Journal of Sports Sciences, 2008; 1557-1565.

11. Gutin B, Barbeau P, Owens S, Lemmon CR, Bauman M, Allison J, Kang HS, Litaker MS. Effects of exercise intensity on cardiovascular fitness, total body composition, and visceral adiposity of obese adolescents. American Journal of Clinical Nutrition, 2002; 75(5): 818-826.

12. Hair JFJ, Anderson RE, Tatham RL, Black WC. Multivariate Data Analysis, (5th Edition). Upper Saddle River, NJ: Prentice Hall, 1998.

13. Hands B, Larkin H, Straker L, Perry M. The relationship among physical activity, motor competence and health-related fitness in 14-year-old adolescents. Scandinavian Journal of Medicine \& Science in Sports, 2009; 19: 655-663.

14. Huang YC, Malina RM. Physical activity and health-related physical fitness in Taiwanese adolescents. Journal of Physiological Anthropology and Applied Human Science, 2002; 21: 11-19.

15. IOM (Institute of Medicine). Fitness Measures and Health Outcomes in Youth. Washington, DC: The National Academies Press, 2012.

16. Jaakkola T, Yli-Piipari S, Huotari P, Watt A, Liukkonen J. Fundamental movement skills and physical fitness as predictors of physical activity: A 6-year follow-up study. Scandinavian Journal of Medicine \& Science in Sports, 2015; doi: 10.1111/sms.12407.

17. Jassen I, Lebance AG. Systematic review of the health benefits of physical activity and fitness in school-aged children and youth. International Journal of Behavioral Nutrition and Physical Activity, 2010; 7(40). Retrieved from http://www.ijbnpa.org/content/7/1/40

18. Katzmarzyk PT, Malina RM, Song TKM, Bouchard C. Physical activity and health fitness in youth: A multivariate analysis. Medicine \& Science in Sports \& Exercise, 1998; 30: 709-714.

19. Malina RM, Bouchard C, Bar-Or O. Growth, maturation, and physical activity. Human Kinetics, Champaign IL, 2004.

20. Malina RM. Physical activity: relationship to growth, maturation, and physical fitness. In: Physical Activity, Fitness, and Health. Bouchard C, Shephard RJ, Stephens T (Eds.). Champaign, IL: Human Kinetics, 1994; 918-930. 
21. Malina RM. Physical activity and fitness: Pathways from childhood to adulthood. American Journal of Human Biology, 2001; 13: 162-172.

22. Meredith MD, Welk GJ. FITNESSGRAM Test Administration Manual (3rd ed). Champaign, IL: Human Kinetics, 2005.

23. Myer GD, Faigenbaum AD, Chu DA, Best TM, Bergeron MF, Hewett TE. Integrative training for children and adolescents: techniques and practices for reducing sports-related injuries and enhancing athletic performance. Physician and Sports Medicine, 2011; 39(1):74-84.

24. Nilsson A, Ekelund U, Yngve A, Sjostrom M. Assessing physical activity among children with accelerometers using different time sampling intervals and placements. Pediatric Exercise Science, 2002; 14(1): 87-96.2.

25. Ortega FB, Ruiz JR, Castillo MJ, Sjostrom M. Physical fitness in childhood and adolescence: A powerful marker of health. International Journal of Obesity, 2008; 32:1-11.

26. Pate RR, O'Neill JR, Lobelo F. The evolving definite of "sedentary". Exercise and Sport Sciences Reviews, 2008; 36(4): 173-178.

27. Pate RR, Pfeiffer KA, Trost SG, Ziegler P, Dowda M. Physical activity among children attending preschools. Pediatrics, 2004; 114: 1256-1263.

28. Powell KE, Caspersen CJ, Koplan JP, Ford ES. Physical activity and chronic diseases. American Journal of Clinical Nutrition, 1989; 49: 999 -1006.

29. Puyau MR, Adolph AL, Vohra FA, Zakeri I, Butte NF. Prediction of activity energy expenditure using accelerometers in children. Medicine \& Science in Sports \& Exercise, 2004; 36(9):1625-31.
30. Rauner A, Flip M, Woll A. The relationship between physical activity, physical fitness and overweight in adolescent: A systematic review of studies published in or after 2000. Pediatrics, 2013; 13:19.

31. Sims J, Scarborough P, Foster C. The Effectiveness of Interventions on Sustained Childhood Physical Activity: A Systematic Review and Meta-Analysis of Controlled Studies. PLoS One, 2015; 10(7): e132935. doi:10.1371/journal.pone.0132935

32. Tabachnick BG, Fidell LS. Using Multivariate Statistics. Boston: Allyn and Bacon. Velicer WF, Fava JL. 1998. Effects of variable and subject sampling on factor pattern recovery. Psychological Methods, 2001; 3(2), 231-251.

33. Trost SG, Loprinzi PD, Moore R, Pfeiffer KR. Comparison of accelerometer cut points for predicting activity intensity in youth. Medicine \& Science in Sports \& Exercise, 2011; 43 (7): 1360-1368.

34. Trost SG, Mciver KL, Pate RR. Conducting accelerometer-based activity assessments in field-based research. Medicine \& Science in Sports \& Exercise, 2005; 37(11): 531-543.

35. US Department of Health and Human Services (USDHSS): 2008 Physical Activity Guidelines for Americans; 2008.

36. Vicente-Rodriguez G, Dorado C, Ara I, Perez-Gomez J, Olmedillas H, Delgado-Guerra S, Calbet JA. Artistic versus rhythmic gymnastics: effects on bone and muscle mass in young girls. International Journal of Sports Medicine, 2007; 28:386-393.

37. Welk GJ, Corbin CB, Dale D. Measurement issues in the assessment of physical activity in children. Research Quarterly for Exercise and Sport, 2000; 71(2): 59-73. 